nation of a flexible bronchoscope. Infect Control Hosp Epidemiol 2009;30(6):550-555.

8. Shimono N, Takuma T, Tsuchimochi N, et al. An outbreak of Pseudomonas aeruginosa infections following thoracic surgeries occurring via the contamination of bronchoscopes and an automatic endoscope reprocessor. I Infect Chemother 2008;14(6): 418-423.

\section{Annual Fluctuation in the Rate of Resistant Bacteria Isolated as an Indicator in the Control of Hospital-Acquired Infections}

To the Editor-The presence of drug-resistant organisms and their variability between hospitals and even within different areas of the same hospital requires a good knowledge of local microbiological epidemiology. The development of antimicrobial stewardship programs (ASPs) in the hospital setting has been encouraged by scientific societies worldwide, ${ }^{1-3}$ and they have proved to be an essential measure in controlling bacterial resistance and antibiotic expenditures. ${ }^{4}$ ASPs establish mechanisms for monitoring and control by the microbiology laboratory by measuring the frequency of isolation of microorganisms with greater importance in the context of nosocomial infection. The aim of this study is to assess the effectiveness of measuring the frequency of isolations per year and the annual fluctuation in the rate of isolation as a working tool in the control of nosocomial infection.

The study was performed in a 790-bed tertiary care academic institution in which microbiology laboratory data about relevant nosocomial isolates are updated monthly. We measure the indicators established by the ASP proposed by the Spanish Society for Infectious Diseases and Clinical Microbiology, ${ }^{3}$ which are expressed in terms not only of absolute and relative frequencies but also of levels of antibiotic resistance and impact on health care units, with special reference to critical patient units. All data are provided to the infection control committee and serve as a tool in updating the local empirical therapy guidelines in the hospital. The indicators that measure the annual frequency levels are methicillinresistant Staphylococcus aureus (MRSA), extended-spectrum $\beta$-lactamase-producing Enterobacteriaceae (ESBL), and multidrug-resistant Acinetobacter baumannii (MDRA). The data are expressed as number of patients, eliminating recurrent strains isolated from the same patient over a period of 4 months.

The first 12-month period began in July 2009. Data were collected monthly, and annual data were obtained until December 2012, which represent 31 points of measurement, which allows observation of trends in the indicator. The most relevant finding was a decrease in patients infected with $A$. baumanni, from 246 MDRA isolates during the first 12month period to $70 \mathrm{MDRA}$ isolates in the most recent 12month period, in agreement with the emergence and control

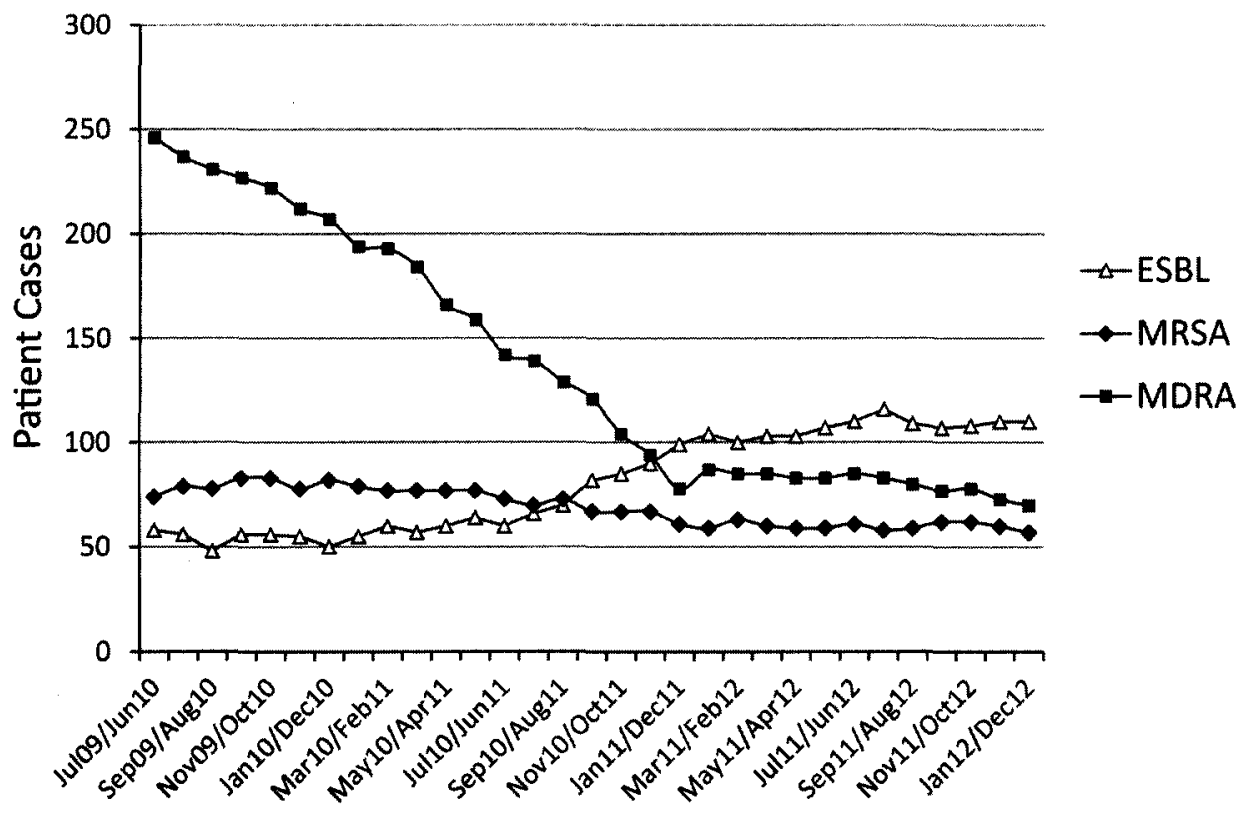

FIGURE 1. Distribution of annual fluctuation in the rate of extended-spectrum $\beta$-lactamase-producing Enterobacteriaceae (ESBL), methicillin-resistant Staphylococcus aureus (MRSA), and multidrug-resistant Acinetobacter baumannii (MDRA) in nosocomial patients (June 2009-December 2012). 
of an outbreak of $A$. baumanni infection in the adult intensive care unit in 2010 that was spread to other ward units in the hospital. However, we observed a sustained increase in the number of patients with an ESBL-positive Enterobacteriaceae isolated (from 58 to 110 patients), and the rate of MRSA isolation remained constant or decreased (from 74 to $57 \mathrm{pa}-$ tients; Figure 1).

Promoting appropriate use of antibiotics and preventing the spread of drug-resistant bacteria are key issues in tackling the public health problem of antimicrobial resistance. ASPs attempt to optimize prescribing of these drugs to benefit both current and future patients. ${ }^{5}$ The introduction of annual fluctuation in the rate of isolation as a measure of the incidence of bacterial isolation from nosocomial patients is an interesting method that provides valuable information easily and is potentially useful for medium-sized hospitals. It is possible to include more important and significant strains in each data cluster obtained and to measure frequency of isolation in the units that require a greater emphasis on infection control. It also provides a projection of the trend and avoids confounding factors, such as seasonal increases due to epidemic outbreaks, different levels of activity throughout the year, and the influence of staff changes that could modify the efficacy of this method in clinical units. In summary, because the local surveillance of antimicrobial resistance is very important, it is necessary that competent professionals regularly process, evaluate, compare, and interpret local data regarding clinically and epidemiologically important patterns of antimicrobial resistance. Intelligible and easy-to-implement outputs of this activity must be routinely distributed to all concerned personnel as appropriate, and the annual fluctuation in the rate of isolation can contribute to this objective.

\section{ACKNOWLEDGMENTS}

Potential conflicts of interest. All authors report no conflicts of interest relevant to this article. All authors submitted the ICMJE Form for Disclosure of Potential Conflicts of Interest, and the conflicts that the editors consider relevant to this article are disclosed here.

Manuel Rodríguez-Iglesias, $\mathrm{MD}, \mathrm{PhD} ;$ Juan Manuel Sanchez-Calvo, PharmD, PhD; ${ }^{1}$ Inmaculada Guerrero-Lozano, PharmD; ${ }^{1}$ Pilar Marin-Casanova, PharmD; ${ }^{1}$ Ana María García-Tapia, PharmD; Fatima Galan-Sanchez, $\mathrm{MD}, \mathrm{PhD}^{1}$

Affiliation: 1. Clinical Microbiology Laboratory, Puerta del Mar University Hospital, University of Cádiz, Cádiz, Spain.

Address correspondence to Manuel Rodríguez-Iglesias, MD, PhD, Clinical Microbiology Laboratory, Puerta del Mar University Hospital, Avenida Ana de Viya, 21, 11009 Cádiz, Spain (manuel.rodrigueziglesias@uca.es).

Infect Control Hosp Epidemiol 2013;34(10):1123-1124
(C) 2013 by The Society for Healthcare Epidemiology of America. All rights reserved. 0899-823X/2013/3410-0019\$15.00. DOI: $10.1086 / 673158$

\section{RE F E R E N CES}

1. Dellit TH, Owens RC, McGowan JE, et al. Infectious Diseases Society of America and the Society for Healthcare Epidemiology of America guidelines for developing an institutional program to enhance antimicrobial stewardship. Clin Infect Dis 2007;15:44.

2. Srinivasan A. Engaging hospitalists in antimicrobial stewardship: the CDC perspective. J Hosp Med 2011;6(suppl 1):S31-S33.

3. Rodriguez-Bano J, Pano-Pardo JR, Alvarez-Rocha L, et al. Programs for optimizing the use of antibiotics (PROA) in Spanish hospitals: GEIHSEIMC, SEFH and SEMPSPH consensus document [in Spanish]. Farm Hosp 2012;36(1):33:e1-e30.

4. Davey $\mathrm{P}, \mathrm{Brown} \mathrm{E}$, Fenelon $\mathrm{L}$, et al. Interventions to improve antibiotic prescribing practices for hospital inpatients. Cochrane Database Syst Rev 2005:CD003543.

5. McGowan JE. Antimicrobial stewardship-the state of the art in 2011: focus on outcome and methods. Infect Control Hosp Epidemiol 2012;33:331-337.

\section{Is Methicillin-Resistant Staphylococcus aureus Colonization Changing? A Study of Academic Health Center Daycare Facilities}

To the Editor-While community-acquired methicillin-resistant Staphylococcus aureus (CA-MRSA) skin infections are prevalent, their mechanism of transmission remains elusive. ${ }^{1}$ Previous studies have documented that infections are common among athletic teams and in schools, daycare centers, and jails. ${ }^{1,2}$ An estimated $1 \%$ of the population is colonized with MRSA, ${ }^{3-6}$ and for healthcare workers the percentage is significantly higher. MRSA transmission from colonized healthcare workers to other family members has also been documented. ${ }^{7}$ We sought to determine the incidence and prevalence of CA-MRSA colonization among people and on surfaces at two university-based daycare centers. This study employed an observational design, in which people and surfaces in a daycare center were swabbed for CA-MRSA.

The study was conducted at two university-based daycare centers in Gainesville, Florida, at the University of Florida. One center serves families of healthcare workers (academic health center); the other serves families of nonclinical faculty and staff (university). Gainesville is a city of 114,000 with a high prevalence of CA-MRSA. ${ }^{8}$ At the time of the study, the facilities served 194 children (103 at the university facility, 91 at the academic health center). A convenience sample of parents, children, and employees of the daycare centers was utilized for the purposes of this study. Eligibility criteria included being able to read and speak English fluently.

Researchers collected demographic information and 3 linked sets of swabs for culture: (1) nose and throat swabs 